\title{
РАСЧЕТ ЦЕНТРА ТЯЖЕСТИ СУДНА В АВТОМАТИЗИРОВАННОМ РЕЖИМЕ В УСЛОВИЯХ ПОГРУЗКИ И МОРЕПЛАВАНИЯ В РЕЖИМЕ РЕАЛЬНОГО ВРЕМЕНИ
}

В статье рассматривается задача автоматизированного расчета метацентрической высоты судна двумя методами: по диаграммам статической и динамической остойчивости, диаграмме Рида. Рассмотрена задача аппроксимации диаграммы статической остойчивости синусоидой. Решена задача двузначности синусоиды, а именно найдена область определения аргумента, необходимая для грании интегрирования функиии. Разработанный алгоритм позволяет значительно сократить время расчета $c$ одновременным повышением точности расчета, а также дает возможность привязки к гироскопу и применить для дальнейшего воплощчения его в контроллерную автоматику, способную отслеживать состояние цеентра тяжести во время погрузки и мореплавания в режиме реального времени, что значительно повысит безопасность эксплуатации судна.

Ключевые слова: метацентрическая высота судна, аппроксимация синусоидой, центр тяжести, автоматизация судовых установок, автоматизированнье системь регулирования, техническая эксплуатациия судов, дискретный контур, гироскоп, компьютеризачия.

Анализ современного состояния проблемы. Для обеспечения безопасности эксплуатации судна во время его погрузки и во время перемещения в морских условиях необходимо правильно и с достаточно высокой точностью уметь рассчитывать метацентрическую высоту. До настоящего времени расчет метацентрической высоты т.е. центра тяжести представлял собой документацию, несвязанную с реальной работой судна. Современный уровень компьютерных технологий позволяет отслеживать состояние диаграммы статической остойчивости как во время погрузки так и во время мореплавания, что в свою очередь позволяет автоматизировать расчет метацентрической высоты в режиме реального времени. В случае опасного смещения центра тяжести компьютерные технологии позволяют подавать звуковой сигнал на самом судне, а также передавать данные через современные связи коммуникации в ближайшие наземные службы безопасности, что должно значительно повысить безопасность работы судна во время его эксплуатации.

Постановка проблемы. Компьютеризировать расчет центра тяжести судна, а также значительно (до нескольких секунд) сократить время расчета с обеспечение высокой точности расчета на протяжении всего времени эксплуатации судна.

Анализ последних исследований и публикаций. Для расчета метацентрической высоты судна необходимо построить диаграммы статической и динамической остойчивости. Диаграмма статической остойчивости отражает колебательный процесс судна и по сути является синусоидой, которую необходимо аппроксимировать.

Цель статьи. Разработать алгоритм автоматизированного расчета метацентрической высоты судна для дальнейшего воплощения его в контроллерную автоматику, способную отслеживать состояние центра тяжести во время погрузки и мореплавания.

Изложение основного материала. Разработано два метода расчета центра тяжести судна:

1. Расчет по диаграммам статической и динамической остойчивости. Применяется в большей степени при погрузке судна;

2. Расчет плеча опрокидывающего момента по диаграмме Рида. Применяется во время мореплавания. 
1. Расчет по диаграммам статической и динамической остойчивости. После распределения груза по танкам и определения осадки судна, а также объемного водоизмещения необходимо определить гидростатические элементы судна по таблицам (из информации по остойчивости и прочности корпуса для капитана). По данным водоизмещения из пантокарены судна (информация по остойчивости прочности корпуса для капитана) строится диаграмма статической и динамической остойчивости. Исходными данными для расчета метацентрической высоты для обоих методов является диаграмма статической остойчивости. Диаграмма статической остойчивости представляет собой дискретную функцию $L=f(\theta)$, где $L$ - метацентрическая высота, $\theta$ - угол крена в градусах и отражает собой колебательный процесс судна как при погрузке так и во время мореплавания по сути является синусоидой, которую необходимо аппроксимировать. Аппроксимирующая функция для кривой статической остойчивости имеет вид $L=a \cdot \sin \left(b \cdot \theta_{R}\right)$, где $\theta_{R}$ - угол крена в радианах. Для определения коэффициентов аппроксимации $a, b$ необходимо применить метод наименьших квадратов. Условная функция имеет вид: $S=\sum_{1}^{n}\left(a \cdot \sin \left(b \cdot \theta_{R}\right)-L\right)^{2}=\min$, тогда

$$
\left\{\frac{\frac{d S}{d a}=2 \cdot \sum_{1}^{n}\left(a \cdot \sin \left(b \cdot \theta_{R}\right)-L\right) \cdot \sin \left(b \cdot \theta_{R}\right)=0}{\frac{d S}{d b}=2 \sum_{1}^{n}\left(a \sin \left(b \cdot \theta_{R}\right)-L\right) \cdot a \cos \left(b \cdot \theta_{R}\right) \cdot \theta_{R}=0}\right\}
$$

В результате получаем систему двух нелинейных уравнений относительно коэффициентов $a, b$. Решить систему возможно методом Ньютона, где:

$$
\left\{\begin{array}{c}
F 1=\sum_{1}^{n}\left(a \cdot \sin \left(b \cdot \theta_{R}\right)-L\right) \cdot \sin \left(b \cdot \theta_{R}\right)=0 \\
F 2=\sum_{1}^{n}\left(a \sin \left(b \cdot \theta_{R}\right)-L\right) \cdot a \cos \left(b \cdot \theta_{R}\right) \cdot \theta_{R}=0
\end{array}\right\} .
$$

Якобиан системы имеет вид:

$$
A 1=\frac{d F 1}{d a} ; B 1=\frac{d F 1}{d b} ; A 2=\frac{d F 2}{d a} ; B 2=\frac{d F 2}{d b} ; C 1=-F 1 ; C 2=-F 2 ;
$$

Коэффициенты А1, В1, С1, А2, В2, С2 являются коэффициентами системы линейных уравнений относительно приращений $d a, d b$.

$$
\left\{\begin{array}{l}
A 1 \cdot d a+B 1 \cdot d b=C 1 \\
A 2 \cdot d a+B 2 \cdot d b=C 2
\end{array}\right\}
$$

Первоначальные значения примем $a=1, b=1 . d a=0 ; d b=0$; Решая систему линейных уравнений относительно приращений $d a, d b$, получим уточненные значения коэффициентов $a, b$. Итерации повторять до тех пор пока не будет выполняться условие: $|a-| d a|| \leq \xi:|b-| d b|| \leq \xi$ В результате некоторых вычислений по методу 1 получили плечи статической и динамической остойчивости и занесли их в табл. 1. 
Плечи статической и динамической остойчивости

\begin{tabular}{|l|r|r|r|r|r|r|r|r|}
\hline${\text { Угол крена, } \boldsymbol{\theta}^{\circ}}^{0,00}$ & 10,00 & 20,00 & 30,00 & 40,00 & 50,00 & 60,00 & 70,00 \\
\hline$L$ стат. & 0,00 & 0,35 & 0,73 & 0,85 & 0,80 & 0,67 & 0,37 & 0,07 \\
\hline$L$ динам. & 0,00 & 0,03 & 0,12 & 0,26 & 0,41 & 0,53 & 0,63 & 0,66 \\
\hline
\end{tabular}

В таблице 2 представлены результаты расчета кривой статической остойчивости $L=f(\theta)$ и аппроксимирующей кривой $L=a \cdot \sin \left(b \cdot \theta_{R}\right)$ в метрах.

В результате расчета коэффициентов аппроксимации получили результат $a=0,8476$; $b=2,6380$. Результаты вычислений отражены в таблице 2.

Таблица 2

Сводная таблица результатов

\begin{tabular}{|c|c|c|c|r|c|}
\hline$\theta^{\circ}$ & $\theta_{R}$ & $L(M)$ & $L_{a}=a \cdot \sin \left(b \cdot \theta_{R}\right)$ & \multicolumn{1}{c|}{$L-L_{a}$} & $\frac{L-L_{a}}{L}$ \\
\hline 0,00 & 0,00 & 0,00 & 0,0000 & 0,0000 & 0,00 \\
\hline 10,00 & 0,17 & 0,35 & 0,3767 & 0,0267 & 0,0763 \\
\hline 20,00 & 0,35 & 0,73 & 0,6749 & $-0,0551$ & $-0,0754$ \\
\hline 30,00 & 0,52 & 0,85 & 0,8325 & $-0,0175$ & $-0,0206$ \\
\hline 35,00 & 0,61 & 0,8 & 0,8166 & 0,0166 & 0,0207 \\
\hline 40,00 & 0,70 & 0,67 & 0,6305 & $-0,0395$ & $-0,0590$ \\
\hline 50,00 & 0,87 & 0,37 & 0,3130 & $-0,0570$ & $-0,1541$ \\
\hline 60,00 & 1,05 & 0,007 & $-0,0697$ & $-0,0767$ & $-0,00$ \\
\hline
\end{tabular}

Графическая интерпретация аппроксимации кривой статической остойчивости $L=f(\theta)$ синусоидой $L=a \cdot \sin \left(b \cdot \theta_{R}\right)$, где $L$ - метацентрическая высота в метрах, $\theta$ - угол крена в градусах, представлена на рис.1.

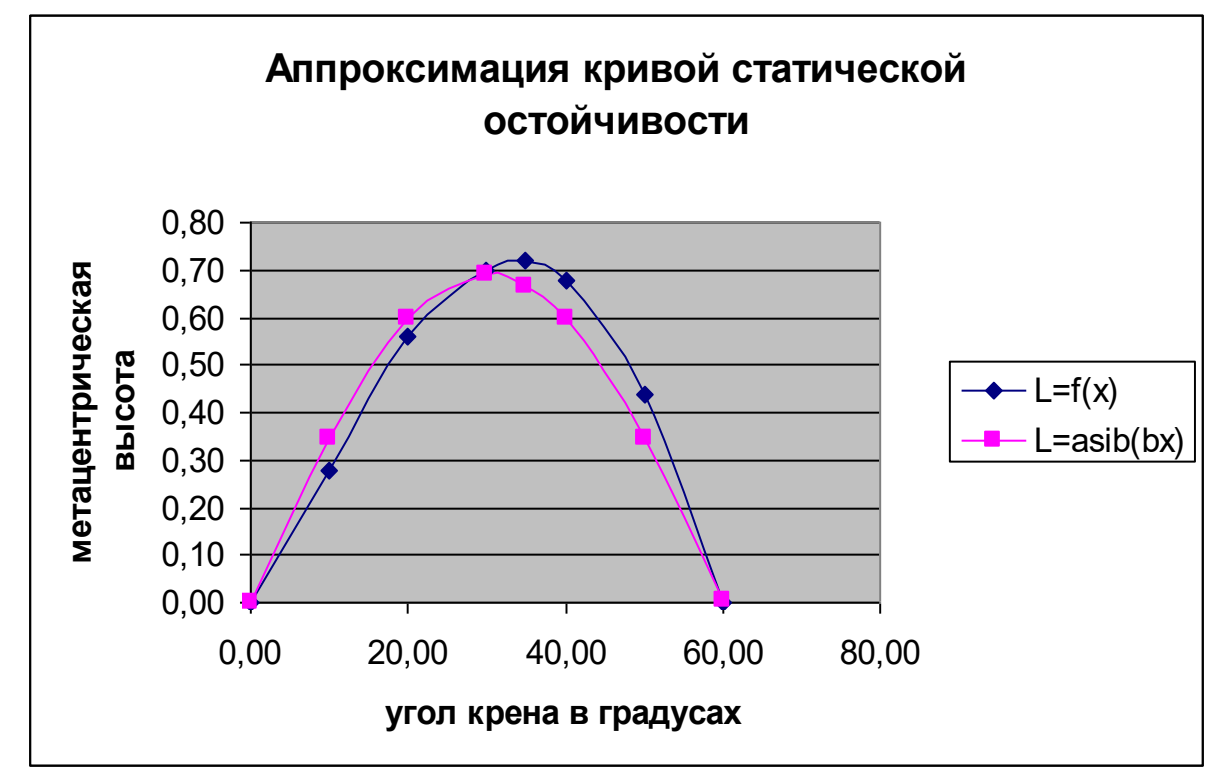

Рис.1. График аппроксимации кривой статической остойчивости $L=f(\theta)$ синусоидой $L=a \cdot \sin \left(b \cdot \theta_{R}\right)$

По результатам статической остойчивости строится кривая динамической остойчивости и по касательной к ней определяется плечо опрокидывающего момента и критерий устойчивости судна. 
Более подробно конкретный пример расчета метацентрической высоты судна рассмотрен на примере танкера «СПЛИТ» для плотности воды $\rho=1,025 \mathrm{~T} / \mathrm{M}^{3}$ в статье [1].

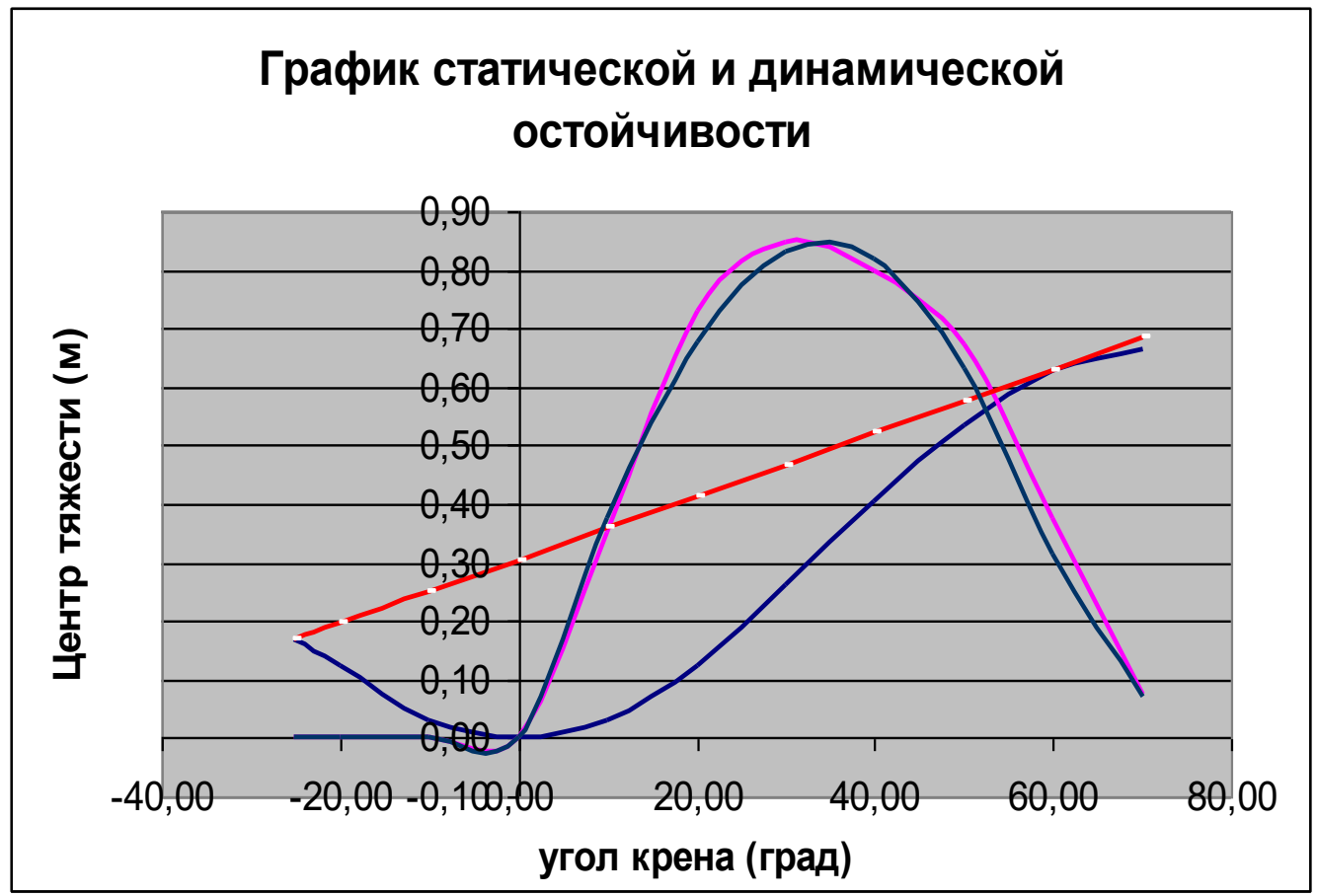

Рис.2. График касательной к кривой динамической остойчивости

\section{2. Расчет плеча опрокидывающего момента по диаграмме Рида.}

Кривая статической остойчивости представлена в таблице 3.

Таблица 3

Кривая статической остойчивости

\begin{tabular}{|l|r|r|r|r|r|r|r|r|}
\hline $\begin{array}{l}\text { Угл крена } \\
\theta^{\text {о }}\end{array}$ & 0,00 & 10,00 & 20,00 & 30,00 & 40,00 & 50,00 & 60,00 & 70,00 \\
\hline$L$ стат. & 0,00 & 0,35 & 0,73 & 0,85 & 0,80 & 0,67 & 0,37 & 0,07 \\
\hline
\end{tabular}

Плечо опрокидывающего момента $L_{\text {опр }}$ определяется по положению, когда площади фигур, заключенные между синусоидой и «подвижной» осью абсцисс равны S1=S2, как показано на рис. 3. Максимальный угол крена $\theta$ по левому борту является переменной. $\mathrm{B}$ данном случае был выбран угол $\theta_{N}=-14^{\circ} . S=\int_{\theta 1}^{\theta 2} a \cdot \sin \left(b \cdot \theta_{R}\right) d \theta_{R}$.

В условиях мореплавания углы ориентации судна, которые являются входными данными для кривой статической остойчивости можно определить в режиме реального времени с помощью гироскопа, связанного с компьютером. 


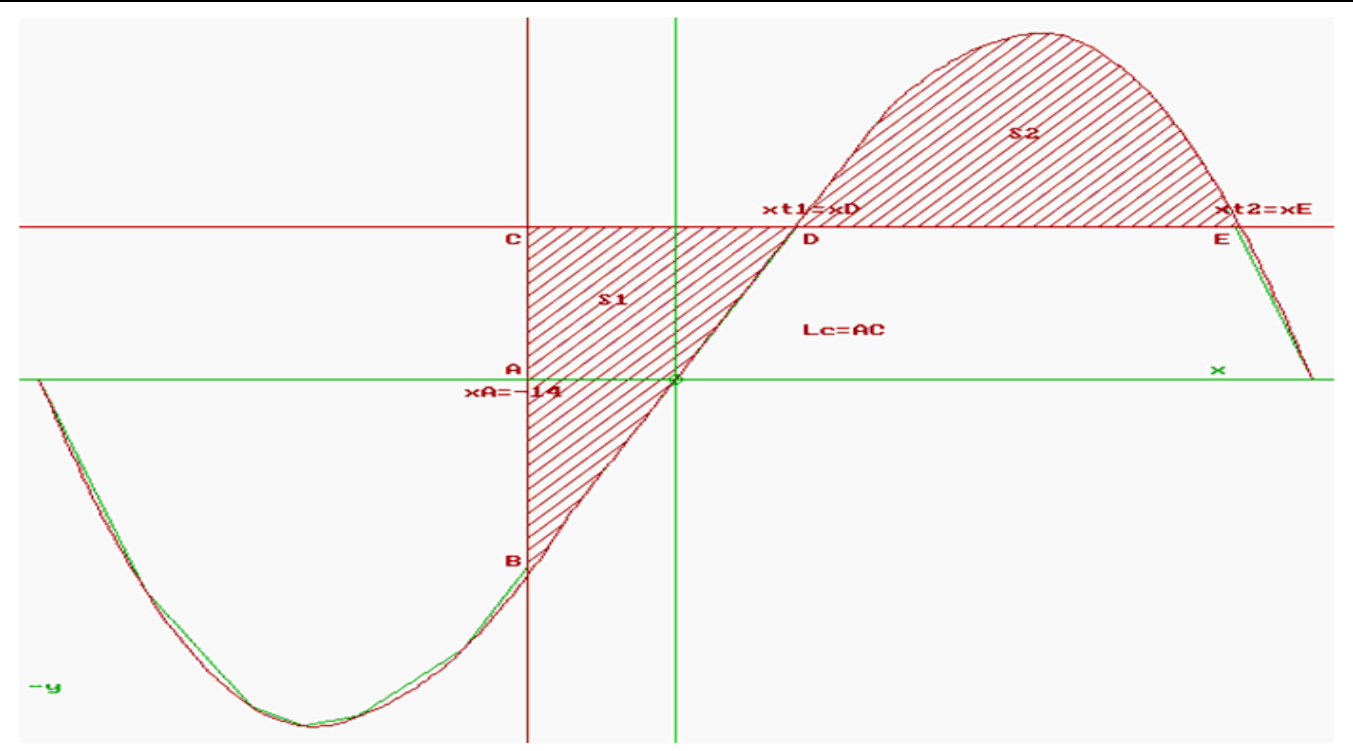

Рис.3. График определения метацентрической высоты судна по диаграмме Рида

Для определения площадей S1 и S2 представляет трудность двузначность синусоиды при одном значении опрокидывающего плеча или центра тяжести. Для решения этой задачи необходимо знать центр симметрии синусоиды в правой ее части. Из уравнения $L=a \cdot \sin \left(b \cdot \theta_{R}\right)$ необходимо определить $\frac{d L}{d \theta_{R}}=a \cdot b \cdot \cos \left(b \cdot \theta_{R}\right)=0$. Из этого следует, что $\left(b \cdot \theta_{R}\right)=\frac{\pi}{2}$, откуда угол в радианах, соответствующий симметрии синусоиды $\theta_{S}=\frac{\pi}{2 b}$. Для данного случая $\theta_{S}=0,6$. Геометрическая интерпретация определения центра симметрии синусоиды представлен на рис.4, откуда следует(рис.3) $x D=\operatorname{Arcsin}\left(a \cdot \sin \left(b \cdot \theta_{R}\right)\right)$, $x E=x D+2(x S-x D)$ или $x E=2 \cdot x S-x D$.

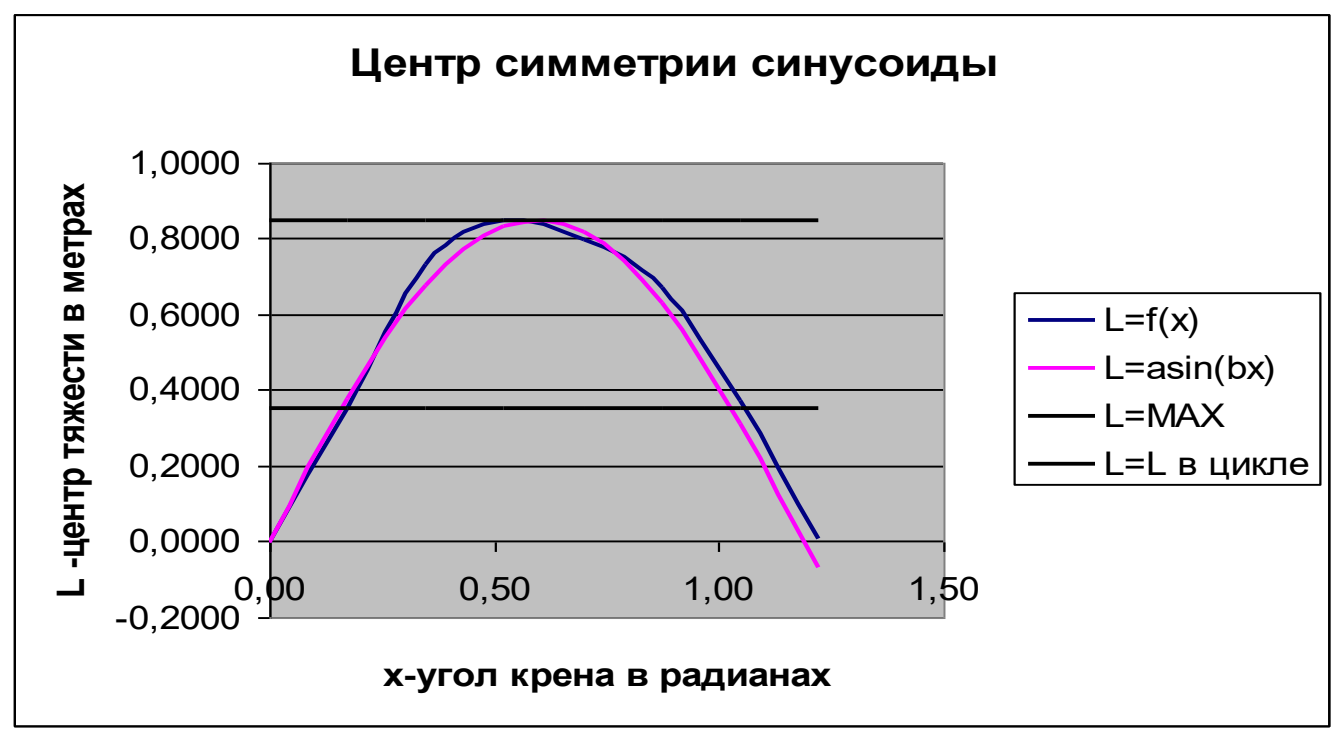

Рис. 4. Пояснение двузначности синусоиды $L=a \cdot \sin \left(b \cdot \theta_{R}\right)$ 
Таблица 4

Область определения угла крена $\theta_{R}$

\begin{tabular}{|c|c|c|c|c|c|c|}
\hline $\begin{array}{c}\text { Угол } \\
\text { крена в } \\
\text { градусах } \\
\theta\end{array}$ & $\begin{array}{c}\text { Угол } \\
\text { крена в } \\
\text { радианах } \\
\theta_{R}\end{array}$ & $\begin{array}{c}\text { Таличное } \\
L(M)\end{array}$ & $\begin{array}{c}L=a \cdot \sin \left(b \cdot \theta_{R}\right) \\
\theta_{R}=x D\end{array}$ & $x D$ & $x E$ & \\
\hline 0,00 & 0,00 & 0,00 & 0,0000 & 0,00 & 1,2 & 0,0 \\
\hline 10,00 & 0,17 & 0,35 & 0,3767 & 0,17 & 1,0163 & 0,3767 \\
\hline 20,00 & 0,35 & 0,73 & 0,6749 & 0,35 & 0,8417 & 0,6749 \\
\hline 30,00 & 0,52 & 0,85 & 0,8325 & 0,52 & 0,6671 & 0,8325 \\
\hline 34,38 & $\theta_{S}=0,6$ & & 0,8476 & 0,6 & 0,6 & 0,8476 \\
\hline
\end{tabular}

Область определения угла крена в радианах $x D<\theta_{R}<x E$ по оси абсцисс показана в таблице 4. Площади $S 1=\int_{x N}^{x D} a \cdot \sin \left(b \cdot \theta_{R}\right)=-\frac{a}{b} \cos (b \cdot x D)+\frac{a}{b} \cos (b \cdot x N)$,

$$
S 2=\int_{x D}^{x E} a \cdot \sin \left(b \cdot \theta_{R}\right)=-\frac{a}{b} \cos (b \cdot x E)+\frac{a}{b} \cos (b \cdot x D) \text {. }
$$

\section{БЛОК-СХЕМА}

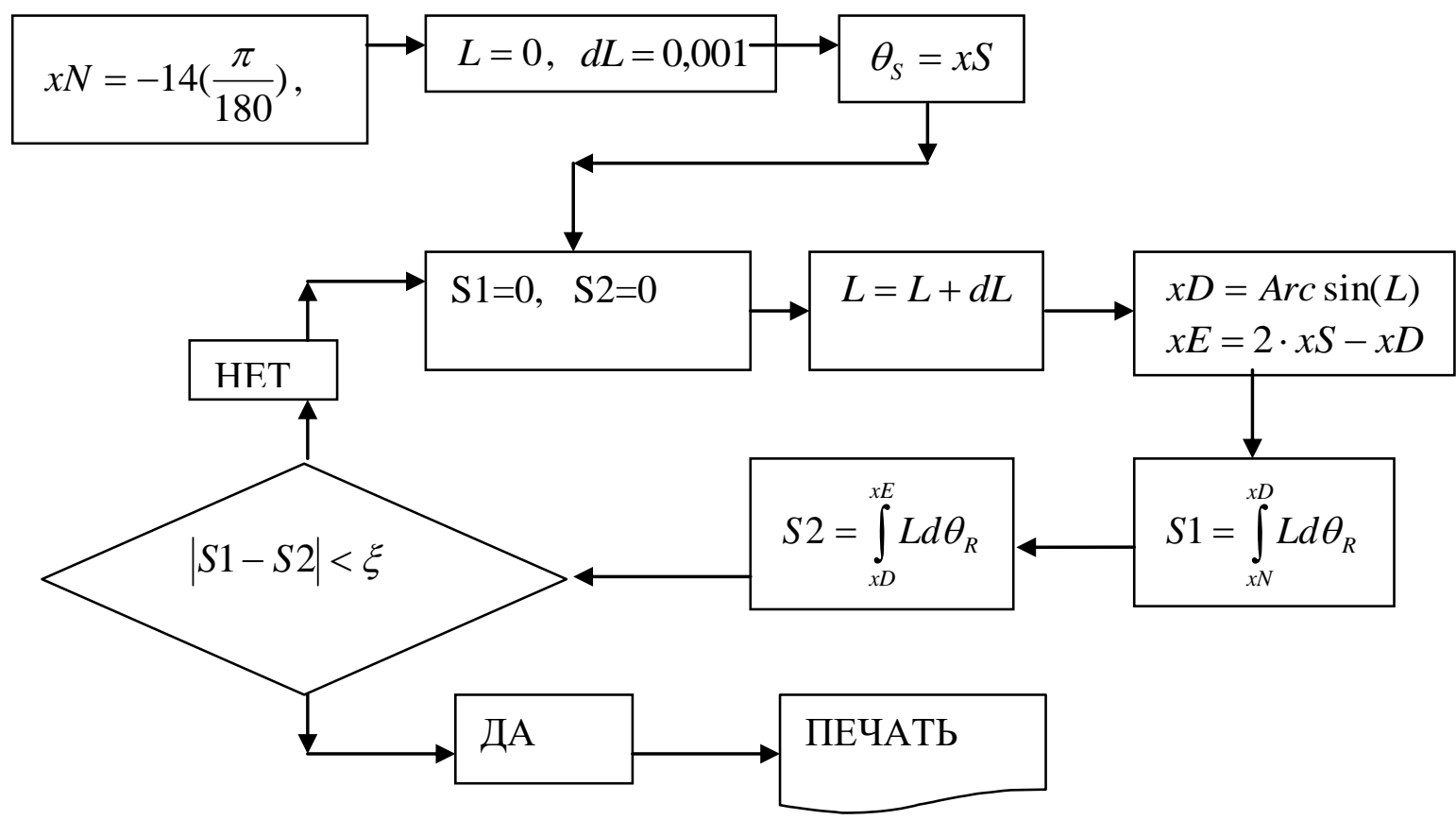

Выводы. Выше был представлен расчет поперечной метацентрической высоты (центра тяжести) судна т.е. по углу крена. Алгоритм расчета пригоден и для продольной устойчивости судна, т.е. по дифференту, а также для любой радиальной плоскости. Выбирая наиболее опасные отклонения есть возможность повысить безопасность эксплуатации судна, как во время погрузки так и во время мореплавания. Следует отметить, что синусоида более точно должна отражать практические условия эксплуатации судна, чем кривая статической остойчивости. Алгоритм расчета метацентрической высоты можно использовать для расчета любого типа судов. 


\section{ЛИТЕРАТУРА}

1. Вільдяєва Л.Н., Блиндарук А.А. Автоматизированный расчет центра тяжести судна при погрузке и в условиях мореплавания с учетом коэффициентов аппроксимации кривых/ Водний транспорт. Збірник наукових праць Київської державної академії водного транспорту. - К.:КДАВТ, - 2015 -№ 2 (23). -С. 217-222.

2. Вагущенко Л.Л., Кошовий А.А. Автоматизовані комплекси судноводіння. Підручник для морських академій // -Київ: КВІЦ. 2001 - 292 с.

3. Толшин В.И., Сизых В.А. Автоматизация судовых энергетических установок Учебник. - 3-е изд. Переработанное и дополненное // - М: Транслит, 2006 - 353 с.

4. Печененко В.И,, Козьминых Г.В. Автоматика регулирования и управления судовых силовых установо //-М.: Транспорт 1973г.

5. Нелепин Р.А. Автоматизация судовых энергетических установок // - Л.: Судостроение 1975 г. -532 c.

6. Шиняев Е.Н. Судовые паровые котлы и их эксплуатация // - М.: Транспорт 1979г.

7. Акимов П.П. Судовые автоматизированные энергетические установки // -M: Транспорт 1980г.

8. Сыромятников В.Ф., Лубочкин Б.И. Автоматическое регулирование судовых паровых котлов // - Л.: Судостроение 1983г.

9. Архангельский В.С. Судовая автоматика // - Л.: Судостроение 1989г.

10. Сизов В.А. Судовая автоматика и контрольно измерительные приборы // М: Транспорт, 1979.

11. Журенко М, А., Таранчук Н.В. Технические средства автоматизации судовых энергетических установок // - Л.: Судостроение 1990г.

12. Балашов Е.П., Григорьев В.Л., Петров Г.А. Микро-и мини -ЭВМ // Л: Энергоатомстроение, Ленинградское отделение 1984 г.-376 с.

13. Вержбицкий В.М. Основы численных методов: Учебник для вузов// -М.: Высш. Шк.,2002.-840c.: ISBN 5-06-004020-8.

\section{REFERENCES}

1. Bildiyiva L.N., Blindaruk A.A. Automated calculation of the center of gravity of the vessel during loading and in the conditions of navigation, taking into account the approximation coefficients of the curves / Water transport. Zbirnik naukovyh prac of the Kiev State Academy of Water Transport.- K.: KDAVT, 2015. № 2 (23). FROM. 217-222.

2. Vaguschenko L.L., Koshoviy A.A. Automation of the shipboard complex. Pidruchnik for marine academies // Kiev: КВІЦ. 2001 - 292 p.

3. Tolshin V.I., Sizykh V.A. Automation of ship power plants. Textbook. - 3rd ed. Revised and supplemented // - M: Translit, 2006 - 353 s.

4. Pechenenko V.I., Kozminykh G.V. Automation of regulation and control of ship power plants // - M .: Transport, 1973.

5. Nelepin R.A. Automation of ship power plants L.: Shipbuilding 1975 - 532 s.

6. Shinyaev E.N. Ship steam boilers and their operation // - M .: Transport 1979.

7. Akimov P.P. Ship automated power plants // -M: Transport 1980.

8. Syromyatnikov V.F., Lubochkin B.I. Automatic regulation of ship steam boilers // - L .: Shipbuilding 1983.

9. Arkhangelsky V.S. Ship automation // - L .: Shipbuilding 1989.

10. Sizov V.A. Ship automation and instrumentation // M: Transport, 1979.

11. Zhurenko M, A., Taranchuk N.V. Technical means of automation of ship power plants // - L .: Shipbuilding 1990 
12. Balashov EP, Grigoryev V.L., Petrov G.A. Micro and mini-computers // L: Energoatomstroyeniye, Leningrad branch of 1984 - 376 p.

13. Verzhbitsky V.M. Fundamentals of numerical methods: Textbook for high schools // -M .: Higher. Shk., 2002. -840 s.

Вільдяєва Л.М.

РОЗРАХУНОК ЦЕНТРА ТЯЖІННЯ СУДНА В АВТОМАТИЗОВАНОМУ РЕЖИМІ ПРИ НАВАНТАЖЕННІ ТА В УМОВАХ МОРЕПЛАВАННЯ У РЕЖІМІ РЕАЛЬНОГО ЧАСУ

У роботі розглянута задача автоматизованого розрахунку метацентричної висоти судна двома методами: по діаграмам статичної та динамічної остійності судна, по діаграмі Ріда. Розглянута задача апроксимачії діаграми статичної остійності синусоїдою. Розв'язана задача двозначності синусоїди тобто знайдена область визначення аргументу, яка необхідна для граниџь інтегрування функиії. Розроблений алгоритм дозволяє значно скоротити час розрахунку з одночасним підвищенням точності розрахунку, а також дає можливість прив'язати до гіроскопу $i$ застосувати для подальшого втілення його $в$ контролерну автоматику, здатну відстежувати стан центра ваги під час навантаження $i$ мореплавання в режимі реального часу, щуо значно підвищить безпеку експлуатації судна.

Ключові слова: ичентр тяжіння судна, математична модель, апроксимація синусоїдою, чисельні методи, автоматизація судових енергетичних установок, автоматизовані системи регулювання, технічна експлуатація суден, дискретний контур, гіроскоп, комп 'ютерізачія.

\section{Vildiaieva L.M.}

\section{AUTOMATED CALCULATION OF CENTER FOR VESSELS LOADING AND UNDER THE CONDITIONS OF NAVIGATION IN REAL TIME}

In the paper the problem of automated calculation of the metacentric height of the vessel is considered by two methods: according to the diagrams of static and dynamic stability of the vessel according to the diagram of Reed. The problem of approximation of the diagram of static stability with a sinusoid is considered. The ambiguity problem of the sinusoid wave is solved - the domain of definition of the argument necessary for the boundaries jf the integration of the function is found. The developed algorithm allows to significantly reduce the calculation time while increasing the accuracy of the calculation, and also makes it possible to applyed the gyroscope and controller automation, which is able to track the state of the center of gravity during loading and navigation in real time, which will significantly increase safety.

Keyword: vessel center of gravity, mathematical model, approximation, numerical methods, automation of marine power plants, automated control systems, technical exploitation of vessels, gyroscope, computerization. 